\title{
Risk for Tuberculosis Disease Among Contacts with Prior Positive Tuberculin Skin Test: A retrospective Cohort Study, New York City
}

\author{
Prabhu P. Gounder, MD, MPH ${ }^{1,2}$, Tiffany G. Harris, PhD, $\mathrm{MS}^{3}$, Holly Anger, MPH ${ }^{3}$, Lisa Trieu, MPH ${ }^{3}$, \\ Jeanne Sullivan Meissner, MPH ${ }^{3}$, Betsy L. Cadwell, MSPH ${ }^{2}$, Elena Shashkina, $P^{3}{ }^{4}$, and \\ Shama D. Ahuja, PhD, MPH
}

'Epidemic Intelligence Service, Centers for Disease Control and Prevention, Atlanta, Georgia; ${ }^{2}$ Scientific Education and Professional Development Program Office, Centers for Disease Control and Prevention, Atlanta, Georgia; ${ }^{3}$ Bureau of Tuberculosis Control, New York City Department of Health and Mental Hygiene, Long Island City, NY, USA; ${ }^{4}$ Public Health Research Institute, Rutgers University, Newark, New Jersey.

BACKGROUND: Patients with prior positive tuberculin skin test (TST) results may benefit from prophylaxis after repeat exposure to infectious tuberculosis (TB).

OBJECTIVE: To evaluate factors associated with active TB disease among persons with prior positive TST results named as contacts of persons with infectious TB.

DESIGN: Population-based retrospective cohort study.

PARTICIPANTS: A total of 2,933 contacts with prior positive TST results recently exposed to infectious TB identified in New York City's TB registry during the period from January 1, 1997 through December 31, 2003.

MAIN MEASUREMENTS: Contacts developing active TB disease $\leq 4$ years after exposure were identified and compared with those who did not, using Poisson regression analysis. Genotyping was performed on selected Mycobacterium tuberculosis-positive isolates.

KEY RESULTS: Among contacts with prior positive TST results, 39 (1.3\%) developed active TB disease $\leq 4$ years after exposure ( $\leq 2$ years: 34 ). Risk factors for contacts that were independently associated with TB were age < 5 years (adjusted prevalence ratio $[\mathrm{aPR}]=19.48$; $95 \%$ confidence interval $[\mathrm{CI}]=7.15-53.09)$, household exposure ( $\mathrm{aPR}=2.60 ; \mathrm{CI}=1.30-5.21)$, exposure to infectious patients (i.e., cavities on chest radiograph, acid-fast bacilli on sputum smear; aPR=1.9 3;CI=1.01-3.71), and exposure to a U.S.-born index patient ( $\mathrm{aPR}=4.04$; $\mathrm{CI}=1.95-8.38)$. Receipt of more than 1 month of treatment for latent TB infection following the current contact investigation was found to be protective $(\mathrm{aPR}=0.27$; $\mathrm{CI}=0.08$ 0.93). Genotype results were concordant with the index patients among 14 of 15 contacts who developed active TB disease and had genotyping results available.

CONCLUSIONS: Concordant genotype results and a high proportion of contacts developing active TB disease within 2 years of exposure indicate that those with prior positive TST results likely developed active TB disease from recent rather than remote infection. Healthcare providers should consider prophylaxis for contacts with prior TB infection, especially young children and close contacts of TB patients (e.g., those with household exposure).

Received August 29, 2014

Revised December 4, 2014

Accepted January 6, 2015

Published online January 21, 2015
KEY WORDS: contact tracing; tuberculosis infection; prevention and control; epidemiology.

J Gen Intern Med 30(6):742-8

DOI: $10.1007 / \mathrm{s} 11606-015-3180-2$

(C) Society of General Internal Medicine 2015

\section{INTRODUCTION}

Contact investigation has been shown to be an effective tuberculosis (TB) control strategy used by public health departments, ${ }^{1,2}$ incorporating goals that include active case findings and completion of treatment for newly infected contacts in order to prevent future TB cases. Approximately half of newly infected contacts develop active TB disease within 2-3 years of infection, ${ }^{3}$ and as such guidelines recommend prophylaxis for contacts who have a new positive test for TB infection (also referred to as latent TB infection). ${ }^{4,5}$ However, contacts identified during a contact investigation may also have a history of a prior positive tuberculin skin test (TST) result; one study found that $10 \%$ of contacts had a prior positive TST. $^{6}$ Until recently, the risk for such individuals to develop active TB disease was unknown, ${ }^{7}$ and guidelines do not make specific treatment recommendations for contacts with a prior positive TST, except to state that decisions to treat must be individualized. ${ }^{1}$

The challenge of evaluating contacts with a prior positive TST result is of particular concern to health departments that serve communities with substantial foreign-born populations, such New York City (NYC). ${ }^{8}$ Foreign-born persons presenting to NYC Department of Health and Mental Hygiene (DOHMH) chest clinics are four times as likely to have a positive TST result as U.S.-born individuals (39.5\% vs. $8.8 \%){ }^{9}$ A recent NYC DOHMH study found that persons with a prior positive TST result were 30 times more likely to have active TB disease identified during contact investigation compared to contacts with negative TST results. ${ }^{7}$ This unexpected finding demonstrated the possibility of new infection among contacts with a prior positive TST result and revealed a potential opportunity to prevent additional cases of active TB disease by identifying and treating those contacts at high risk for active TB disease.

We conducted a retrospective cohort study to determine contact and corresponding index patient characteristics that 
were associated with developing active TB disease among contacts with a prior positive TST and to investigate whether active TB disease among contacts with a prior positive TST might be attributable to the exposure under current investigation rather than previous exposure.

\section{METHODS}

\section{Study Population}

This analysis is part of a larger study evaluating the effectiveness of contact investigation in reducing the incidence of TB. As described in the previous study, 36,606 contacts of 5,731 persons with infectious TB disease from 1997-2003 were identified from the NYC TB registry (Fig. 1). ${ }^{7}$ An index patient was defined as the person with confirmed infectious TB reported to the NYC DOHMH. Contacts were persons named during contact investigation of an infectious TB patient who was exposed during the infectious period. TB patients were classified as having infectious TB if their sputum was either acid-fast bacilli (AFB) smear-positive or culturepositive for Mycobacterium tuberculosis complex. The infectious period was defined as 3 months before the index patient started anti-TB treatment, based on guidelines for contact investigations established by the NYC DOHMH and the Centers for Disease Control and Prevention (CDC). ${ }^{1,10}$ Contacts were excluded from analysis if they lived or had relocated outside NYC, died during contact investigation, had an unknown date of birth, had health care-associated exposure, were exposed to an index patient $<5$ years of age, had multidrug-resistant $\mathrm{TB}$, or had received treatment for active TB disease $\leq 1$ year before the index patient's diagnosis. For persons identified as a contact multiple times during the study period, the most recent exposure and evaluation outcome was used in the analysis. After exclusions, 30,561 contacts of 5,182 persons with infectious TB remained. As described previously, all contacts were cross-referenced with the TB registry from January 1, 1997 through December 31, 2007 , in order to identify those who either had active TB disease at the time of contact investigation or subsequently developed active TB disease within 4 years of the index patient's date of diagnosis. Follow-up time was restricted to 4 years in order to maintain consistency with the parent study. ${ }^{7}$

The final study population included the 2,933 (10\%) contacts (of 1,740 persons with infectious TB) with evidence of a prior positive TST result at the time of contact investigation. Contacts were classified as having a prior positive TST if they had a positive TST documented in the TB registry prior to the index patient's infectious period, provided documentation of a prior positive TST result during the contact investigation, or provided a history compatible with a prior diagnosis of TB infection (e.g., previous treatment for TB infection). In addition to the exclusions made by the parent study (described previously), the current study also excluded contacts with a history of prior active TB disease.

This study was approved by the NYC DOHMH Institutional Review Board and was given a non-humansubject research determination by the CDC.

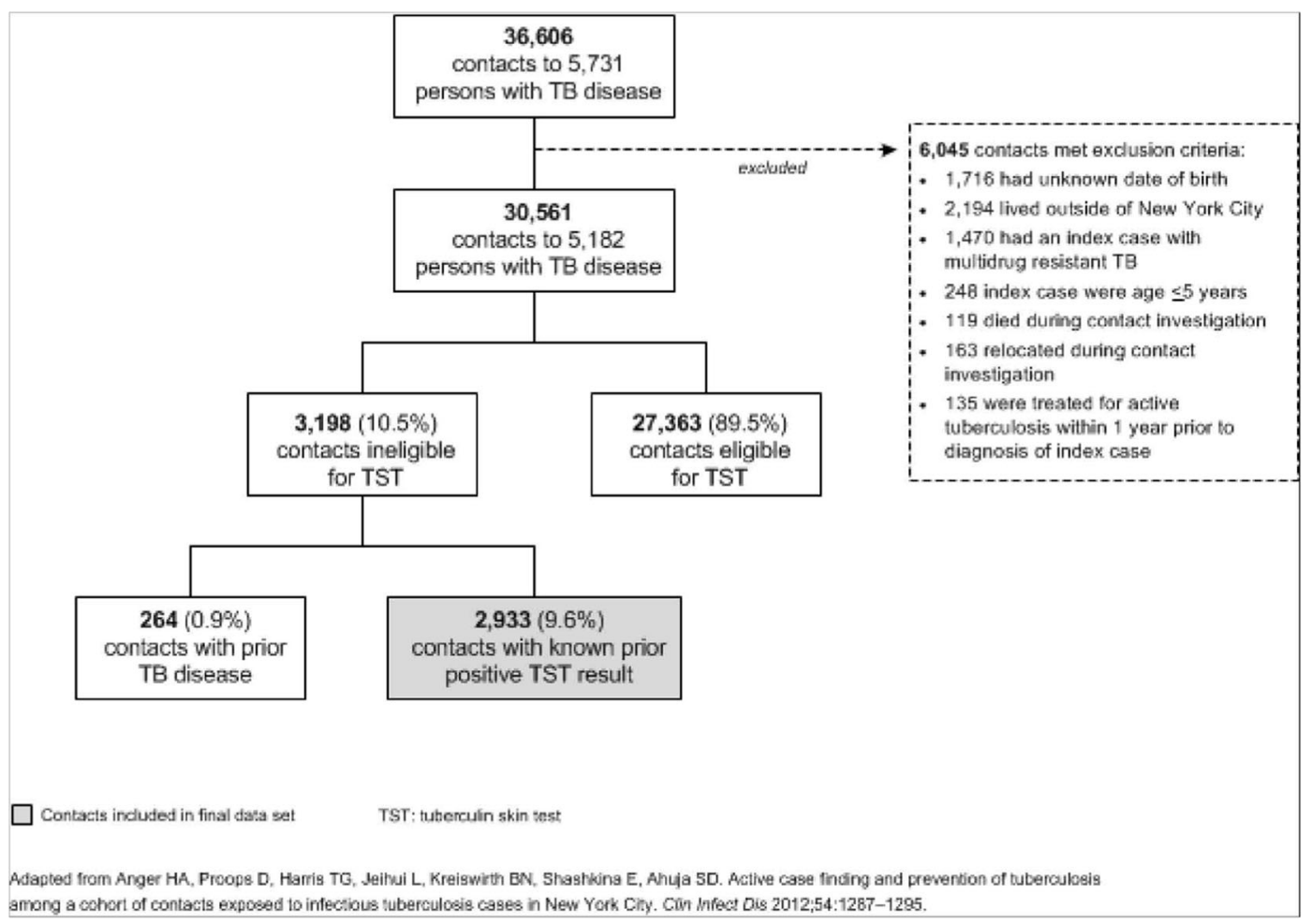

Figure 1 Study population of contacts to persons with tuberculosis (TB) disease, New York City, 1997-2003. 


\section{Data Analysis and Statistical Methods}

Demographic, clinical, laboratory, treatment, and exposure information for index patients and contacts was obtained from the NYC TB registry. The number of contacts developing active TB disease $\leq 4$ years after the index patient's diagnosis was divided by the total number of contacts with a prior positive TST result in order to determine the period prevalence for active TB disease. Risk factors among contacts for developing active TB disease were identified using Poisson regression analysis with generalized estimating equations to calculate unadjusted and adjusted prevalence ratios (PRs and aPRs, respectively) and $95 \%$ confidence intervals (CIs) for contact and corresponding index patient characteristics. We estimated prevalence ratios rather than risk ratios, which are based on incidence, because we did not distinguish between contacts having active TB disease at the time of the contact investigation (i.e., a prevalent TB case) from contacts who developed active TB disease after the contact investigation (i.e., an incident case). To assess characteristics independently associated with active TB disease, the regression model included the following variables (determined a priori), along with any contact or index patient variables significantly $(P<0.05)$ associated with active TB disease in the unadjusted analysis: age at time of contact investigation ( $<5$ versus $\geq 5$ years), household versus non-household setting of exposure to index patient, treatment for TB infection as part of the contact investigation (defined as $>1$ month of chemoprophylaxis for TB infection), and exposure to a more infectious index patient (defined as cavities on chest radiograph or presence of AFB on sputum smear). Although place of birth and human immunodeficiency virus (HIV) status are recognized as important factors for developing active TB disease, these variables were not included in the final multivariable regression model due to substantial missing data.

Where available, genotype results among contacts who developed active TB disease and the corresponding index case patient (case-pair) were reviewed to assess whether transmission between the index patient and contact was plausible. Since 2001, genotyping has been performed in NYC on all initial M. tuberculosis culture-positive isolates using IS6110 restriction fragment length polymorphism (RFLP) analysis and spacer oligonucleotide typing (spoligotyping). Before 2001, genotyping was performed on isolates from selected TB patients. ${ }^{11}$ For purposes of this analysis, genotype results were defined as concordant between members of a case-pair if RFLP and spoligotype patterns matched exactly or if the RFLP patterns matched exactly but spoligotype results were unavailable. To account for the possibility of spontaneous bacterial mutation events before or after transmission, genotype results were also considered concordant if no more than a one-band RFLP difference existed between case-pair isolates (i.e., near-match) that had exactly matching spoligotype patterns and when subsequent review by the genotyping laboratory confirmed the plausibility of direct transmission on the basis of RFLP pattern similarity. ${ }^{12,13}$

\section{RESULTS}

Among 2,933 contacts with a prior positive TST result identified in NYC, a total of 39 (1.3\%) developed active TB disease $\leq 4$ years after the index patient's diagnosis date. Of these, 34 (87\%) developed active TB disease $\leq 2$ years after the index patient's diagnosis, and 23 (59\%) developed active TB disease $<9$ months after the index patient's diagnosis.

Among contacts with a prior positive TST result (Table 1), $60 \%$ were female and $80 \%$ were aged $18-64$ years at the time of contact investigation. Nearly half (45\%) of contacts were foreign-born; however, the country of birth was unknown for one-third of the contacts. HIV status was unknown for $90 \%$ of contacts, $9 \%$ were reported as HIV-uninfected, and $1 \%$ were reported as HIV-infected. Approximately half of the contacts (48\%) were exposed in a household setting, whereas the others $(52 \%)$ were exposed in congregate settings (e.g., schools or work). Of the 642 (22\%) contacts who received at least 1 month of treatment for TB infection after evaluation during the contact investigation, $574(89 \%)$ used isoniazid and $61(10 \%)$ used rifampin. Among contacts initiating TB infection treatment, 353 (55\%) completed the full treatment course (53\% who received isoniazid, $72 \%$ who received rifampin).

Of the 39 contacts with a prior positive TST results who developed active TB disease, 11 (28\%) were foreign-born (Table 1). Of the eight foreign-born contacts with a known date of entry into the U.S., four developed active TB disease $\leq 5$ years after entry (including one at the time of entry), whereas four had active TB disease $>13$ years after entry (data not shown).

In terms of index patient characteristics (Table 1), $62 \%$ percent of contacts were exposed to a foreign-born index patient, and $20 \%$ of contacts were known to be exposed to an HIV-infected index patient. Among the 32 contact-index patient pairs where country of birth was known for both parties, the country of birth was the same in $24(75 \%)$ pairs (data not shown). With regard to social risk factors, $14 \%$ of contacts were exposed to an index patient with a history of illegal drug use, and $7 \%$ were exposed to an index patient with a history of homelessness. Lastly, $33 \%$ of contacts were exposed to an index patient with evidence of increased infectiousness.

Risk factors associated with active TB disease among contacts with a prior positive TST result, calculated using univariate analysis, included age $<5$ years, U.S. birth, household exposure to the index patient, HIV infection, exposure to a U.S.-born index patient, and exposure to an index patient with a history of homelessness (Table 1). Receipt of $\geq 1$ month of treatment for TB infection as part of the contact investigation protected contacts from developing active TB disease, even if the treatment was incomplete. Of the three contacts who received $\geq 1$ month of treatment for latent tuberculosis infection (LTBI) and who developed active TB disease, one completed a full course of treatment. 
Table 1 Contact and Index Patient Characteristics Associated with Developing Tuberculosis (TB) Disease Among Contacts with Prior Positive Tuberculin Skin Test (+TST) Results-New York City, 1997-2003

\begin{tabular}{|c|c|c|c|c|c|c|}
\hline & \multicolumn{2}{|c|}{$\begin{array}{l}\text { Contacts with a } \\
\text { prior + TST result }\end{array}$} & \multicolumn{2}{|c|}{$\begin{array}{l}\text { TB disease } \\
\text { among contacts }\end{array}$} & \multirow[b]{2}{*}{ Crude PR (95 \% CI) } & \multirow[b]{2}{*}{ Adjusted PR (95\% CI) ${ }^{\dagger}$} \\
\hline & No. & $(\%)$ & No. & $(\%)^{*}$ & & \\
\hline \multicolumn{7}{|l|}{ Contact characteristics } \\
\hline Total & 2,933 & & 39 & (1) & & \\
\hline Male sex & 1,167 & $(40)$ & 21 & (54) & $1.77(0.97-3.23)$ & \\
\hline \multicolumn{7}{|c|}{ Age (years) at TB exposure } \\
\hline $0-4$ & 23 & (1) & 4 & (10) & $13.34(4.71-37.74)$ & \\
\hline $5-17$ & 328 & (11) & 3 & (8) & $0.70(0.21-2.37)$ & \\
\hline $18-44$ & 1,457 & $(50)$ & 19 & $(50)$ & Ref. & \\
\hline $45-64$ & 884 & (30) & 13 & (33) & $1.13(0.55-2.31)$ & \\
\hline$\geq 65^{+}$ & 241 & (8) & 0 & 0 & - & \\
\hline \multicolumn{7}{|c|}{ Age $<5$ years at TB exposure } \\
\hline Yes & 23 & (1) & 4 & $(10)$ & $14.46(5.40-38.73)$ & 19.48 (7.15-53.09) \\
\hline No & 2,910 & (99) & 35 & (90) & Ref. & \\
\hline \multicolumn{7}{|l|}{ Birth in the United States } \\
\hline Yes & 632 & $(22)$ & 23 & (59) & $4.35(2.11-8.96)$ & \\
\hline No & 1,314 & (45) & 11 & (28) & Ref. & \\
\hline Unknown & 987 & (34) & 5 & (13) & $0.61(0.21-1.73)$ & \\
\hline \multicolumn{7}{|l|}{ Setting of TB exposure } \\
\hline Household & 1,398 & (48) & 25 & (64) & $1.96(1.01-3.79)$ & $2.60(1.30-5.21)$ \\
\hline Non-household ${ }^{\S}$ & 1,535 & $(52)$ & 14 & (36) & Ref. & Ref. \\
\hline \multicolumn{7}{|l|}{ HIV status } \\
\hline HIV-uninfected & 257 & (9) & 4 & (10) & Ref. & \\
\hline HIV-infected & 42 & (1) & 5 & (13) & $4.90(1.33-18.00)$ & \\
\hline Unknown status & 2,634 & $(90)$ & 30 & (77) & $0.59(0.23-1.50)$ & \\
\hline \multicolumn{7}{|c|}{ Received $\geq 1$ month of treatment for TB infection" } \\
\hline No & 2,242 & (76) & 36 & $(92)$ & Ref. & Ref. \\
\hline Yes & 642 & (22) & 3 & $(8)$ & $0.29(0.09-0.92)$ & $0.27(0.08-0.93)$ \\
\hline Unknown & 49 & $(2)$ & 0 & 0 & - & \\
\hline \multicolumn{7}{|c|}{ Index patient characteristics } \\
\hline Male sex & 1,704 & $(58)$ & 25 & (64) & $1.29(0.67-2.48)$ & \\
\hline Age (years) & & & & & & \\
\hline $5-17$ & 109 & (4) & 4 & (10) & $2.36(0.83-6.69)$ & \\
\hline $18-44$ & 1,541 & (53) & 24 & (62) & Ref. & \\
\hline $45-64$ & 734 & (25) & 11 & (28) & $0.96(0.46-2.02)$ & \\
\hline$>65^{\ddagger}$ & 549 & (19) & 0 & 0 & - & \\
\hline \multicolumn{7}{|c|}{ Birth in the United States } \\
\hline Yes & 1,102 & (38) & 27 & (69) & $4.07(2.03-8.17)$ & $4.04(1.95-8.38)$ \\
\hline No & 1,827 & $(62)$ & 11 & (28) & Ref. & Ref. \\
\hline More infectious ${ }^{\dagger \dagger}$ & 967 & (33) & 18 & (46) & $1.74(0.92-3.29)$ & $1.93(1.01-3.71)$ \\
\hline \multicolumn{7}{|l|}{ HIV status } \\
\hline HIV-uninfected & 1,588 & $(54)$ & 23 & $(59)$ & Ref. & \\
\hline HIV-infected & 566 & (20) & 11 & (28) & $1.34(0.65-2.78)$ & \\
\hline Unknown status & 779 & (27) & 5 & (13) & $0.44(0.17-1.16)$ & \\
\hline History of drug use ${ }^{* *}$ & 397 & (14) & 8 & (21) & $1.74(0.80-3.79)$ & \\
\hline History of homelessness & 216 & (7) & 7 & (18) & $2.75(1.24-6.09)$ & $2.09(0.90-4.88)$ \\
\hline
\end{tabular}

Abbreviations: CI confidence interval, HIV human immunodeficiency virus, Ref. referent group, PR prevalence ratio, - not calculated

${ }^{*}$ Cases occurring $\leq 4$ years after index patient's date of diagnosis

${ }^{*}$ The adjusted Poisson regression model included the variables determined a priori, along with any contact variables statistically significantly associated with TB disease in the unadjusted analysis.

${ }^{7}$ Observations from categories with 0 TB cases were excluded from regression model for that variable.

$\S$ Refers to non-household congregate settings such as school or workplace

"Treatment for TB infection after contact investigation

** Any illicit substance use ever, not including alcohol or tobacco; status unknown for 37 contacts, including two TB patients

${ }^{H C}$ Cavitary lesion on chest radiograph or sputum smear-positive for acid-fast bacilli

In addition to the a priori-determined characteristics, the multivariate model included exposure to an index patient with a history of homelessness and exposure to a U.S.-born index patient (Table 1). The following risk factors were independently associated with developing active TB disease among contacts: age $<5$ years $(\mathrm{aPR}=19.48 ; \mathrm{CI}=7.15-53.09)$, exposure in a household setting $(\mathrm{aPR}=2.60 ; \mathrm{CI}=1.30-5.21)$, exposure to a more infectious index patient $(\mathrm{aPR}=1.93 ; \mathrm{CI}=1.01-3.71)$, and exposure to a U.S.-born index patient $(\mathrm{aPR}=4.04 ; \mathrm{CI}=1.95-8.38)$. The protective effect of receiving $\geq 1$ month of treatment for
TB infection remained significant $(\mathrm{aPR}=0.27 ; \mathrm{CI}=0.08-0.93$ ). Contacts who developed active TB disease within 9 months of their index patient's diagnosis may not have had an opportunity to initiate treatment for TB infection, and so a sensitivity analysis was conducted to further evaluate this protective effect. When analysis was limited to the 16 contacts who developed active TB disease after 9 months, the protective effect of receiving $\geq 1$ month of treatment for TB infection was no longer statistically significant in either the unadjusted $(\mathrm{PR}=0.81 ; \mathrm{CI}=0.23-2.83)$ or adjusted $(\mathrm{aPR}=1.08 ; \mathrm{CI}=0.32$ 3.62) analyses. 
Table 2 Genotype Results for Contacts Developing Tuberculosis Disease and for Corresponding Index Patients-New York City, 1997-2003*

\begin{tabular}{|c|c|c|c|c|c|c|}
\hline Contact & $\begin{array}{l}\text { Date of } \\
\text { diagnosis }\end{array}$ & $\begin{array}{l}\text { Months since index } \\
\text { patient diagnosis }\end{array}$ & $\begin{array}{l}\text { Household } \\
\text { exposure }\end{array}$ & $\begin{array}{l}\text { Exact RFLP match } \\
\text { between patients }\end{array}$ & $\begin{array}{l}\text { Exact spoligotype match } \\
\text { between patients } \uparrow\end{array}$ & $\begin{array}{l}\text { Plausible } \\
\text { transmission }\end{array}$ \\
\hline 1 & Apr 1998 & 2.6 & No & Yes & Not performed & Yes \\
\hline 2 & Jul 1998 & 15.7 & No & Yes & Not performed & Yes \\
\hline 3 & Mar 2000 & 1.9 & No & Yes & Not performed & Yes \\
\hline 4 & Jul 2000 & 1.5 & Yes & Yes & Yes & Yes \\
\hline 5 & Jan 2001 & 10.5 & Yes & No & Yes & Yes \\
\hline 6 & Apr 2001 & 4.8 & Yes & Yes & Yes & Yes \\
\hline 7 & Sep 2001 & 4.8 & Yes & Yes & Yes & Yes \\
\hline 8 & Feb 2002 & 28.5 & No & No & Not performed & No \\
\hline 9 & Apr 2002 & 0.2 & Yes & No & Yes & Yes \\
\hline 10 & Nov 2002 & 1.6 & Yes & Yes & Yes & Yes \\
\hline 11 & Apr 2003 & 0.4 & Yes & Yes & Yes & Yes \\
\hline 12 & Nov 2003 & 1.3 & Yes & Yes & Yes & Yes \\
\hline 13 & Jun 2004 & 13.5 & No & Yes & Yes & Yes \\
\hline 14 & Sep 2004 & 37.3 & Yes & No & Yes & Yes \\
\hline 15 & Jul 2005 & 42.3 & Yes & No & Yes & Yes \\
\hline
\end{tabular}

Abbreviations: RFLPrestriction fragment length polymorphism, spoligotypespacer oligonucleotide typing

* Among contacts with known prior positive tuberculin skin test result and index patients with a positive culture for Mycobacterium tuberculosis and RFLP result * "Not performed" indicates spoligotype not performed for either the contact or the index patient.

* Transmission is considered plausible if the genotypes of corresponding index and contact case-pairs were concordant. Concordance is defined as casepairs having an exact RFLP and exact spoligotype match, an exact RFLP match but spoligotype was not performed, or exact spoligotype match with no more than a one-band RFLP difference, and subsequent non-blinded review by the genotyping laboratory confirmed plausibility of direct transmission based on RFLP pattern similarity.

Genotype results were available for $15(39 \%)$ contacts who developed active TB disease and their corresponding index patients (Table 2); 14 (93\%) case-pairs had concordant genotypes (three pairs by RFLP alone; seven pairs with matching RFLP and spoligotype; and four pairs with matching spoligotype and near-matching RFLP). The three case-pairs that were concordant by RFLP alone had patterns with $\geq 10$ bands. Among the 14 case-pairs with concordant genotypes, $10(71 \%)$ contacts had household exposure to the index patient. For the discordant case-pair, the contact was a Haitian-born worksite contact of a Bangladesh-born index patient.

\section{DISCUSSION}

This study demonstrated that, for contacts with prior positive TST results, the risk for developing active TB disease $\leq 4$ years after exposure to an index patient was $1.3 \%$, and $87 \%$ of those contacts developed active TB disease within 2 years of exposure. Contacts who were $<5$ years of age at the time of exposure, had household exposure, or were exposed to a more infectious index patient were at increased risk of developing active TB disease. Contacts who received $\geq 1$ month of treatment for TB infection during the contact investigation were protected against developing active disease. However, this protective effect may be overestimated, as prevalent cases (i.e., those who developed active disease $<9$ months after exposure) who did not have an opportunity to initiate treatment for TB infection were included among contacts not receiving treatment. When the analysis was limited to only incident cases (i.e., those developing active disease $>9$ months after exposure), the protective effect of treatment was no longer statistically significant, albeit the sample size was small. Our finding supporting the beneficial effect of initiating treatment is consistent with results in the parent study. ${ }^{7}$ Further research is necessary to evaluate the magnitude of this protective effect.

Current recommendations assume that persons with prior positive TST results developed TB infection prior to the current contact investigation; however, guidelines acknowledge that risk for active TB disease after repeat exposure is undetermined. ${ }^{1,2}$ Our findings indicate that active TB disease among contacts with prior positive TST results likely resulted from recent exposure rather than reactivation of remote infection. The majority of contacts experiencing active TB disease were exposed to the index patient in a household setting and developed TB disease within 2 years of exposure, consistent with evidence that the period of highest risk for developing active TB disease is shortly after exposure. ${ }^{3,14}$ Additionally, genotype results were concordant for $93 \%$ of the case-pairs with genotyping data. Matching genotype results - especially in the presence of strong epidemiologic links - is routinely interpreted in research and in public health practice as evidence of transmission. Genotype concordance between casepairs does not definitively prove transmission, because they might have shared a common exposure or been independently infected by different persons with the same $M$. tuberculosis strain. (Multiple case-pairs in our study received a diagnosis of active TB disease within months of each other, for example, which may be indicative of prevalent TB acquired from a common source; alternately, the index patient might have been identified first despite earlier disease onset in the contact patient).

These results support current NYC DOHMH guidelines outlining criteria for determining contacts who should be referred for medical evaluation. Specifically, NYC guidelines recommend referring all contacts $<5$ years of age and those 
with HIV infection for evaluation and treatment of TB infection, regardless of TST status, as they are known to be at high risk for active TB. ${ }^{5}$ For contacts with a prior positive TST, NYC guidelines recommend medical evaluation for individuals who are symptomatic, for those who have substantial exposure ( $\geq 8$ hours/week) to a person with highly infectious pulmonary or laryngeal $\mathrm{TB}$, or when definitive transmission is observed. In addition, our results indicate that all contacts with a prior positive TST who were exposed in a household setting should be considered for medical evaluation and treatment, regardless of prior TST status.

This study has certain limitations. It is a retrospective analysis of data collected for public health practice; information on certain TB risk factors (e.g., HIV and country of birth) was unknown for a substantial proportion of contacts. In addition, we did not compare risk factors for active TB disease between contacts with prior positive TST results and those with negative TST results. Therefore, it is possible that differences in the prevalence of risk factors between the two groups could partially account for the elevated risk for active TB disease observed among contacts with a prior positive TST result. Third, active TB disease occurring among contacts who moved away from NYC after the contact investigation or who reported a history of TB infection but whose TST status was unknown or undocumented at the time of contact investigation were excluded from the present analysis, possibly resulting in a lower prevalence estimate. Genotyping data was only available for a subset of index case and contact case-pairs, and because genotyping was not universal before 2001, the proportion of cases with missing results was not evenly distributed over the study period. Finally, a substantial proportion of contacts were born outside the U.S. and may have received the bacillus Calmette-Guérin (BCG) vaccine, which could have resulted in a false-positive TST result. It is important to note that $\mathrm{CDC}$ guidelines recommend interpreting a positive TST in a foreign-born or BCGvaccinated person as evidence of $\mathrm{TB}$ infection, and to evaluate/treat accordingly. ${ }^{1}$

The study also has considerable strengths. To our knowledge, this is the first study evaluating the risk for active TB disease among contacts with a prior positive TST result. NYC is one of the few jurisdictions with an electronic registry that has systematically collected information on both TB index patients and contacts for decades. The substantial number of contacts and index patients in our study provided the statistical power needed to identify potential risk factors among this cohort. The availability of genotype data enabled us to assess plausibility of transmission between a subset of contact case and index case-pairs.

Our results are consistent with previous studies demonstrating that the risk for active TB disease among contacts with a prior positive TST result is elevated compared to those with a negative TST result. ${ }^{15,16}$ It is not likely that all contacts with prior positive TST results acquired a new TB infection, which could explain their lower risk for active TB disease compared with contacts with a new positive TST. Therefore, it is possible that the risk for active TB disease among contacts with a new TB infection is similar, regardless of TST status. Because existing diagnostic tests cannot distinguish active TB infection caused by a prior exposure from a new infection caused by recent exposure, physicians should consider prophylaxis for contacts with prior TB infection, especially young children and those with close contact to TB patients (e.g., household exposure).

Acknowledgments: Contributors: The authors thank Julie Magri, MD, MPH, Centers for Disease Control and Prevention, for her input in the design of the study and for reviewing drafts of this manuscript; Douglas Proops, MD, MPH, New York City Department of Health and Mental Hygiene, for critical review of the manuscript; and the Bureau of TB Control staff who conducted the contact investigations.

Funders: This work was supported by the New York City Department of Health and Mental Hygiene, the Bureau of Tuberculosis Control program funds, and in-kind personnel support from the Centers for Disease Control and Prevention. The findings and conclusions in this report are those of the authors, and do not necessarily represent the official position of the Centers for Disease Control and Prevention.

Prior presentations: Preliminary results from this analysis were presented at the 2012 National TB Workshop, June 12-14, 2012, in a poster entitled "Risk for Tuberculosis Disease Among Contacts with Preexisting Latent Tuberculosis Infection—New York City, 1997-2003."

Conflict of Interest: None of the authors have any relevant conflicts to declare.

Corresponding Author: Shama D. Ahuja, PhD, MPH; Bureau of Tuberculosis Control, New York City Department of Health and Mental Hygiene, 42-09 28th Street, 21st floor, CN72-B, Long Island City, NY 11101, USA (e-mail: sahuja@health.nyc.gov).

\section{REFERENCES}

1. Guidelines for the investigation of contacts of persons with infectious tuberculosis. Recommendations from the National Tuberculosis Controllers Association and CDC. MMWR Recomm Rep. 2005;54(RR15): $1-47$.

2. American Thoracic Society/Centers for Disease Control and Prevention/ Infectious Diseases Society of America: controlling tuberculosis in the United States. Am J Respir Crit Care Med. 2005;172(9):1169227. doi:10.1164/rccm.2508001.

3. Ferebee SH. Controlled chemoprophylaxis trials in tuberculosis. A general review. Bibl Tuberc. 1970;26:28-106.

4. Targeted tuberculin testing and treatment of latent tuberculosis infection. American Thoracic Society. MMWR Recomm Rep. 2000;49(RR-6): 1-51.

5. New York City Department of Health and Mental Hygiene. Tuberculosis Clinical Policies and Protocols. 4th ed: New York City Department of Health and Mental Hygiene; March 2008.

6. Marks SM, Taylor Z, Qualls NL, Shrestha-Kuwahara RJ, Wilce MA, Nguyen CH. Outcomes of contact investigations of infectious tuberculosis patients. Am J Respir Crit Care Med. 2000;162(6):2033-8.

7. Anger HA, Proops D, Harris TG, Jeihui L, Kreiswirth BN, Shashkina E, et al. Active case finding and prevention of tuberculosis among a cohort of contacts exposed to infectious tuberculosis cases in New York City. CID. 2012:54(9): 1287-95.

8. U.S. Census Bureau. State \& County Quick Facts. http://quickfacts. census.gov/qfd/states/36/3651000.html. Accessed June 15, 20122012.

9. Li J, Munsiff SS, Agerton TB. Prevalence of tuberculin skin test positivity in clinical population in New York City. J Immigr Minor Health Center Minor Public Health. 2010;12(6):816-22. doi:10.1007/s10903-008-9204-9.

10. Munsiff SS, Nielsen D, Fujiwara P. Clinical policies and protocols. 2004. 
11. Clark CM, Driver CR, Munsiff SS, Driscoll JR, Kreiswirth BN, Zhao B, et al. Universal genotyping in tuberculosis control program, New York City, 20012003. Emerg Infect Dis. 2006;12(5):719-24. doi:10.3201/eid1205.050446.

12. Warren RM, van der Spuy GD, Richardson M, Beyers N, Booysen C, Behr MA, et al. Evolution of the IS6110-based restriction fragment length polymorphism pattern during the transmission of Mycobacterium tuberculosis. J Clin Microbiol. 2002;40(4):1277-82.

13. Warren RM, van der Spuy GD, Richardson M, Beyers N, Borgdorff MW, Behr MA, et al. Calculation of the stability of the IS6110 banding pattern in patients with persistent Mycobacterium tuberculosis disease. J Clin Microbiol. 2002;40(5):1705-8.
14. Cronin WA, Golub JE, Lathan MJ, Mukasa LN, Hooper N, Razeq JH, et al. Molecular epidemiology of tuberculosis in a low- to moderateincidence state: are contact investigations enough? Emerg Infect Dis. 2002;8(11):1271-9. doi:10.3201/eid0811.020261.

15. Andrews JR, Noubary F, Walensky RP, Cerda R, Losina E, Horsburgh CR. Risk of progression to active tuberculosis following reinfection with mycobacterium tuberculosis. Clin Infect Dis. 2012;54(6):784-91. doi:10.1093/cid/cir951.

16. Israel HL, Hetherington HW, Ord JG. A study of tuberculosis among students of nursing. J Am Med Assoc. 1941;117(10):839-44. doi:10.1001/ jama.1941.02820360021007. 